\title{
A NOTE ON MULTIVALENCE OF FUNCTIONS OF BOUNDED INDEX
}

\author{
GERD H. FRICKE
}

\begin{abstract}
This paper examines the relationship between the concept of bounded index and the radius of univalence, respectively $p$-valence, of entire functions and their derivatives at arbitrary points in the plane.
\end{abstract}

1. Introduction. An entire function $f(z)$ is of bounded index if there exists an integer $N$, independent of $z$, such that

$$
\max _{0 \leqq l \leqq N}\left\{\frac{\left|f^{(l)}(z)\right|}{l !}\right\} \geqq \frac{\left|f^{(n)}(z)\right|}{n !} \text { for all } n .
$$

The least such integer $N$ is called the index of $f(z)$. The concept of functions of bounded index was first introduced by Lepson [4].

For an entire function $f$ and a complex number $w$ let $r(w, f)$ denote the radius of univalence of $f(z+w)$. Let $R_{N}(w)=R_{N}(w, f)=$ $\max _{0 \leqq i \leqq N}\left\{r\left(w, f^{(i)}\right)\right\}$. Then we prove the following results.

THEOREM 1. Let $f$ be an entire function. Then $f^{\prime}$ is of bounded index if and only if there exist an integer $N>0$ and a constant $\delta>0$ such that $R_{N}(w) \geqq \delta$ for all $w \in C$, i.e. for any point in the plane, for one of the first $N$ derivatives has radius of univalence of at least $\delta$.

THEOREM 2. Let $f$ be an entire function. Then $f^{\prime}$ is of bounded index if and only if there exists an integer $p>0$ such that $f$ is p-valent in any disc of radius 1 .

2. Proof of Theorem 1. " $\Rightarrow$ " Let $f^{\prime}$ be of bounded index. Then, by [2, Theorem 2], $f^{\prime}(\alpha z)$ is of bounded index for any $\alpha \in C$. Let $N$ be the index of $f^{\prime}(2 z)$ and, for $w \in C$, let $f(z)=\sum_{n=0}^{\infty} a_{n}(z-w)^{n}$.

Hence, there exists an integer $k, 0 \leqq k \leqq N$, such that

$\left|a_{k+1}\right| \geqq(k+1)^{-1}(k+1+j) 2^{j}\left|a_{k+1+j}\right| \geqq 2^{j}\left|a_{k+1+j}\right| \quad$ for $j=1,2, \cdots$.

Presented to the Society, November 20, 1972; received by the editors November 28 , 1972.

AMS (MOS) subject classifications (1970). Primary 30A66; Secondary 30A64.

Key words and phrases. Functions of bounded index, univalent, $p$-valent, exponential type.

(c) American Mathematical Society 1973 
Now,

$$
f^{(k)}(z)=\sum_{n=k}^{\infty} n(n-1) \cdots(n-k+1) a_{n}(z-w)^{n-k}=\sum_{j=0}^{\infty} b_{j}(z-w)^{j} .
$$

Therefore, for $j=1,2, \cdots$,

Thus,

$$
\begin{aligned}
\frac{\left|b_{j+1}\right|}{\left|b_{1}\right|} & =\frac{(k+1+j)(k+j) \cdots(j+2)}{(k+1) !} \frac{\left|a_{k+1+j}\right|}{\left|a_{k+1}\right|} \\
& \leqq(j+1)^{k} \frac{\left|a_{k+1+j}\right|}{\left|a_{k+1}\right|} \leqq \frac{(j+1)^{k}}{2^{j}}
\end{aligned}
$$

$$
\sum_{n=2}^{\infty} n\left|b_{n}\right| \leqq \sum_{j=1}^{\infty} \frac{(j+1)^{k+1}}{2^{j}}\left|b_{1}\right| \leqq\left|b_{1}\right| \sum_{j=1}^{\infty}(j+1)^{N+1} 2^{-j}=\left|b_{1}\right| B,
$$

where $B>0$ is a constant independent of $w$. It is well known that $\sum_{n=2}^{\infty} n\left|c_{n}\right|<1$ implies $g(z)=z+\sum_{n=2}^{\infty} c_{n} z^{n}$ is starlike univalent in $|z|<1$, (compare [1]). Therefore, $f^{(k)}(z)=\sum_{j=0}^{\infty} b_{j}(z-w)^{j}$ is univalent in $|z-w|<$ $1 / B$.

" $\Leftarrow$ " Choose $N>0$ and $\delta>0$ such that $R_{N}(w) \geqq \delta$ for all $w \in C$. Then, given $w \in C$, there exists an integer $k \leqq N$ such that $f^{(k)}(z)=\sum_{j=0}^{\infty} b_{j}(z-w)^{j}$ is univalent in $|z-w|<\delta$. Obviously $b_{1} \neq 0$ and therefore

$$
g(z)=\sum_{n=1}^{\infty} \frac{b_{n}}{b_{1}} \delta^{n-1} z^{n}=\sum_{n=1}^{\infty} c_{n} z^{n}
$$

is univalent in $|z|<1$ with $c_{1}=1$. It is well known that then $\left|c_{n}\right|<e n$ for $n=2,3, \cdots$, (see [3, Theorem 1.6]). Hence, $\left|b_{1}\right| \geqq\left(\left|b_{n}\right| / e n\right) \delta^{n-1}$ for $n=$ $1,2, \cdots$. Now, if $f(z)=\sum_{t=0}^{\infty} a_{t}(z-w)^{t}$ then

$$
\left|a_{k+1}\right|=\frac{\left|b_{1}\right|}{(k+1) !} \geqq \frac{\left|b_{n}\right|}{(k+1) !} \frac{\delta^{n-1}}{e n} \geqq\left|a_{k+n}\right| \frac{\delta^{n-1}}{e n} \text { for } n=1,2, \cdots \text {. }
$$

Hence, $f^{\prime}(z / T)$ is of index not exceeding $N$ for $T$ sufficiently large and thus $f^{\prime}(z)$ is of bounded index. Q.E.D.

3. Proof of Theorem 2. " $\Rightarrow$ " Let $f^{\prime}$ be of index $N$, let $b$ be a root of $f(z)=w$, and let $f(z)=w+\sum_{n=1}^{\infty} a_{n}(z-b)^{n}$. Since $f^{\prime}$ is of index $N$, there exists an integer $k$ with $1 \leqq k \leqq N+1$ such that $\left|a_{k}\right| \geqq\left|a_{j}\right|$ for $j=1,2, \cdots$. Let $P(z)=\sum_{n=1}^{k}\left(a_{n} / a_{k}\right) z^{n}$. Then $P(z)=\prod_{j=1}^{k}\left(z-c_{j}\right)$ for some $c_{j} \in C$. Let $T=\{4(N+1)\}^{N+1}$. Then there exists a constant $r$ with $(2 k T)^{-1} \leqq r \leqq 1 / T$ such that for $z$ with $|z|=r$ we have $\left|z-c_{j}\right| \geqq(2 k T)^{-1}$ for $j=1, \cdots, k$. Thus $|P(z)| \geqq(1 / 2 k T)^{k}$ for all $|z|=r$. Now, for $|z|=r$,

$$
\left|\sum_{n=k+1}^{\infty} \frac{a_{n}}{a_{k}} z^{n}\right| \leqq \sum_{n=k+1}^{\infty}\left|\frac{a_{n}}{a} z^{n}\right| \leqq \sum_{n=k+1}^{\infty}|z|^{n} \leqq \sum_{n=k+1}^{\infty} T^{-n}<\frac{2}{T^{k+1}} .
$$


Therefore,

$$
|P(z)| \geqq(2 k T)^{-k} \geqq \frac{2}{T^{k+1}}>\left|\sum_{n=k+1}^{\infty} \frac{a_{n}}{a_{k}} z^{n}\right| \text { for all }|z|=r .
$$

By Rouché's theorem, $\sum_{n=1}^{\infty}\left(a_{n} / a_{k}\right) z^{n}$ has the same number of zeros in $|z|<r$ as $P(z)$. Hence $\sum_{n=1}^{\infty} a_{n} z^{n}$ has at most $k$ zeros in $|z|<(2 k T)^{-1}$ and $f(z)=w$ has at most $k$ solutions in $|z-b|<(2 k T)^{-1}$. In general, $f(z)$ is at most $(N+1)$-valent in $|z-\gamma|<\{4(N+1) T\}^{-1}$ for all $\gamma \in C$. Thus there exists $p \geqq N+1$ such that $f$ is $p$-valent in any disc of radius 1 .

" $\Leftarrow$ " Let $f(z)=\sum_{n=0}^{\infty} a_{n}(z-w)^{n}$ be $p$-valent in $|z-w|<1$. Without loss of generality we may assume $a_{0}=0$. Then, by [2, Theorem 3.5] we have, for $n=1,2, \cdots,\left|a_{n}\right|<A(p) \max _{1 \leq \nu \leq p}\left\{\left|a_{v}\right|\right\} n^{2 p-1}$, where $A(p)$ depends only on $p$. Thus, in general,

$$
\frac{\left|f^{(n)}(z)\right|}{n !}<A(p) n^{2 p-1} \max _{1 \leqq \nu \leqq p}\left\{\frac{\left|f^{(\nu)}(z)\right|}{\nu !}\right\} \text { for } n=1,2, \cdots .
$$

Hence, $f^{\prime}(z / T)$ is of index $p$ for $T$ sufficiently large and therefore $f^{\prime}(z)$ is of bounded index. Q.E.D.

\section{REFERENCES}

1. J. Clunie and F. R. Keogh, On starlike and convex schlicht functions, J. London Math. Soc. 35 (1960), 229-233. MR 22 \#1682.

2. G. H. Fricke, A characterization of functions of bounded index, Indian J. Math. (to appear).

3. W. K. Hayman, Multivalent functions, Cambridge Tracts in Math. and Math. Phys., no. 48, Cambridge Univ. Press, Cambridge, 1958. MR 21 \#7302.

4. B. Lepson, Differential equations of infinite order, hyperdirichlet series and entire functions of bounded index, Entire Functions and Related Parts of Analysis, Proc. Sympos. Pure Math. 11, Amer. Math. Soc., Providence, R.I., 1968, pp. 298-307. MR 38 \#6069.

Department of Mathematics, University of Kentucky, Lexington, Kentucky 40506 